\title{
Cerebral and cardiac toxicity of a small dose of lignocaine
}

\author{
Herman O. Klein, Itzhak Jutrin, and Elieser Kaplinsky \\ From The Cardiology Service and Department of Medicine A., Meir General Hospital, Kfar-Saba, and Sackler \\ Medical School of the University of Tel-Aviv, Israel
}

\begin{abstract}
$A$ patient is reported in whom an intravenous bolus injection of $50 \mathrm{mg}$ lignocaine produced extreme sinus bradycardia (rate I4 to $20 / \mathrm{min}$ ). Only 2 patients with such a response to a similarly small dose have been previously reported, but these reports indicate clearly that careful electrocardiographic monitoring is advisable when lignocaine is administered.
\end{abstract}

Lignocaine enjoys wide recognition as the drug of choice for the acute treatment of ventricular arrhythmias. Recent reports have called for caution in its use in patients who are elderly, or in those who have severe congestive heart failure or hepatic disease, inferior wall myocardial infarction, and those with dysfunction of the sinus node (Cheng and Wadhwa, r973; Zelis, 1972). We wish to report here the extreme sensitivity (both cerebral and cardiac) to a relatively small dose $(50 \mathrm{mg})$ of lignocaine in a patient in whom none of the above pathological states were present.

\section{Case report}

A 62-year-old diabetic man was admitted to hospital on 9 August 1973, with ventricular tachycardia. Six years before admission, he had suffered an acute inferior myocardial infarction. A second myocardial infarct in I970 had been complicated by transient ventricular tachycardia. He was subsequently maintained on quinidine sulphate $200 \mathrm{mg} 4$ times a day. Two weeks before the present admission, he had been admitted because of similar tachycardia and chest pains; cardioversion was successfully performed, no myocardial infarction was detected, and he was discharged after a few days.

On the day of the present admission, the patient was brought to the emergency room with chest pains and palpitations. The heart rate was $150 / \mathrm{min}$, and the blood pressure was $160 / 90 \mathrm{mmHg}(2 \mathrm{I} .2 / 12.0 \mathrm{kPa})$. The chest was clear on auscultation, there were no murmurs, and there was no hepatic enlargement or tenderness. Blood urea, electrolytes, calcium, phosphorus, and liver enzymes (SGOT, SGPT, alkaline phosphatase) were normal. Blood glucose was $150 \mathrm{mg} / 100 \mathrm{ml}(8.33 \mathrm{mmol} / \mathrm{l})$. An electrocardiogram showed a tachycardia at a rate of I50 beats per minute and widened QRS complexes. The rhythm was erroneously interpreted by the housestaff as being supraventricular tachycardia with aberration. The only treatment given during the first day was three oral doses of $250 \mathrm{mg}$ procainamide. His chest pains subsided without any specific treatment and the blood pressure and pulse remained unchanged.

An electrocardiogram (Fig. I) recorded on the second day revealed fusion beats and a biphasic pattern of the QRS complex in VI, features that suggested that the tachycardia was ventricular in origin. The patient was therefore given an intravenous bolus of $50 \mathrm{mg}$ lignocaine over 2 minutes. The tachycardia promptly abated and was replaced by normal sinus rhythm (at a rate of 75/ $\mathrm{min}$ ) as the last few milligrams of drug were given.

However, within three to four minutes, the patient had, in quick succession, three generalized grand mal convulsions which lasted a few seconds each. He then received $2.0 \mathrm{mg}$ diazepam intravenously, after which he remained drowsy. The respiratory rate remained good. An electrocardiogram recorded after the last convulsion showed normal sinus rhythm at a rate of 75 per minute. A few seconds later, the sinus rate was noted to slow considerably and was then replaced by a slow AV junctional rhythm (Fig. 2A) with a rate of $43 / \mathrm{min}$. As this AV junctional rhythm also slowed, an extremely slow and unstable sinus rhythm became re-established (Fig. $2 \mathrm{~B}-\mathrm{E}$ ) with a rate as slow as $\mathrm{I} 4 / \mathrm{min}$ (Fig. $2 \mathrm{E}$ ). Because of the onset of extreme sinus bradycardia cardiac massage was performed although spontaneous respiration persisted, and isoprenaline was injected intravenously. As a result, AV junctional rhythm with a rate of $90 / \mathrm{min}$, and then normal SA nodal rhythm were re-established. The patient recovered fully. Serial myocardial enzymes 
$\underline{A}$

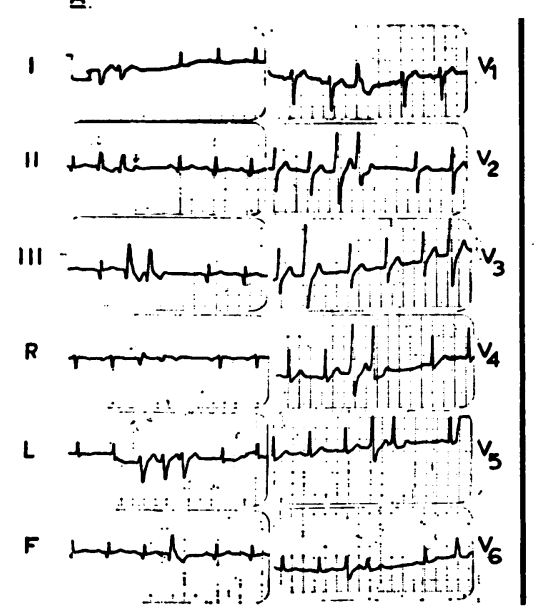

旦

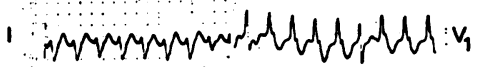

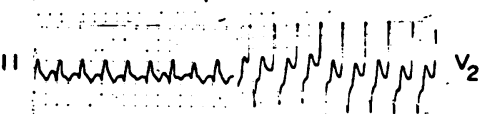

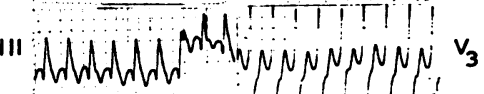

a

$\mathbf{R}$

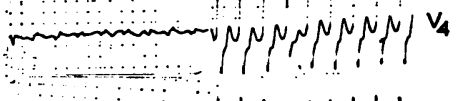

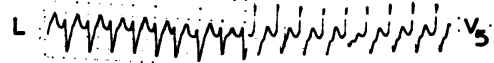

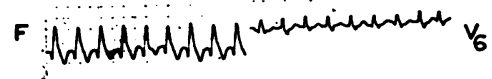

FIG. I $A$ ) Tracing obtained during sinus rhythm with frequent ventricular premature beats. $B)$ Tachycardia with a rate of $150 / \mathrm{min}$. The $Q R S$ complexes are similar to the ventricular premature beats in A. This feature and the appearance of fusion beats (e.g. first beat in aVR, penultimate beat in aVL, 6th beat in VI, 4th beat in V4, 5th beat in $V_{5}$ ) indicate that the tachycardia is most probably of ventricular origin.

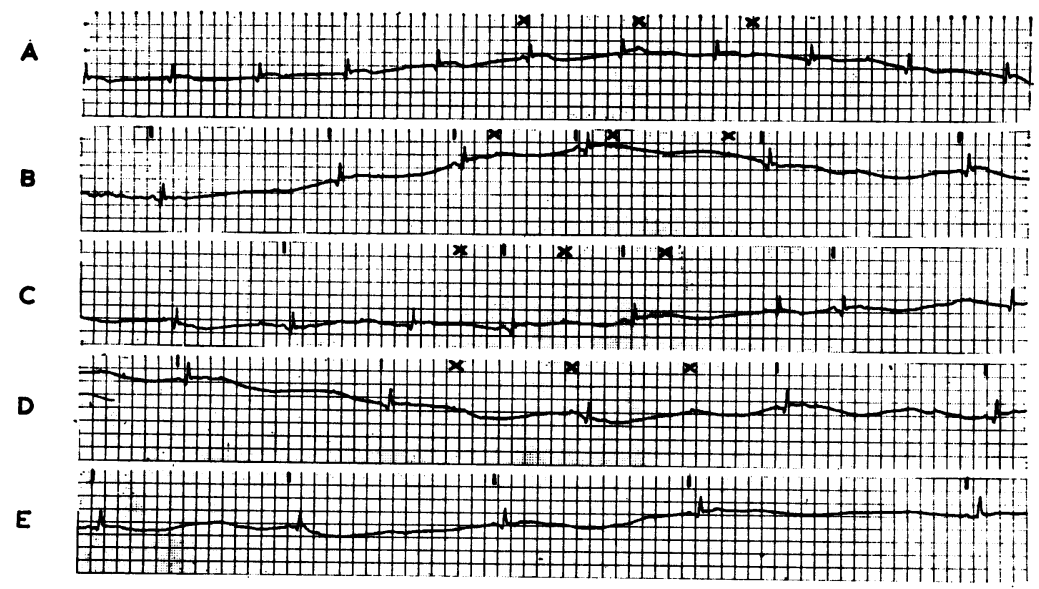

FIG. 2 Effect of lignocaine on the electrocardiogram. A) Slow AV junctional rhythm. Appearance of deflections of the baseline (marked $x$ ) which may represent artefacts caused by laboured breathing. $B$ ) and $C$ ) Further slowing of $A V$ junctional rhythm and reappearance of an extremely slow $S A$ rhythm (marked I). The 3rd, 6th, and 8th $Q R S$ in $C$ represent escape $A V$ junctional beats. $D$ ) and $E$ ) Further slowing of $S A$ nodal rhythm. Baseline deflections are no longer visible (lead III: ECG speed $-25 \mathrm{~mm} / \mathrm{s}$ ). 
remained normal. A careful investigation (including inspection of the vials and the quantity of drug withdrawn for the treatment of the patient) failed to reveal any error in dosage of the lignocaine solution. A I2-lead electrocardiogram obtained subsequently during normal sinus rhythm with frequent ventricular premature ectopic beats (Fig. IA) showed that the configuration of these ventricular beats was clearly similar to that of the QRS complexes during the tachycardia (Fig. IB). This feature further confirmed the correctness of the impression of ventricular tachycardia.

The patient is being further investigated with regard to the possibility of a ventricular aneurysm being present and related to the occurrence of the ventricular tachycardia.

\section{Discussion}

Lignocaine is probably the safest drug available for the acute treatment of ventricular arrhythmias. This is due to the relative absence of side effects, as well as to the short duration of these side effects when they do occur. Yet, two recent cases and one editorial have called attention to the possibility of severe sinus bradycardia or standstill with relatively small doses of lignocaine (Lippestad and Forfang, 1971; Wood, 1971; Zelis, 1972). As far as we are aware this is the third reported case in the English literature of unusual sensitivity to a relatively small dose of the drug. Another report has recently documented the occurrence of sinoatrial standstill after lignocaine (Cheng and Wadhwa, I973); however, the dose used was at the upper limit of the recommended range, the patient was 88 years old, and he was recovering from severe acute pulmonary oedema. An additional patient reported by FlenstedJensen and Sandøe (1969) developed asystole after the injection of $150 \mathrm{mg}$ of lignocaine. He also had had a large acute anterior wall infarction and had received antazoline. One other patient developed a similar reaction while receiving lignocaine by continuous infusion and after having been treated with quinidine sulphate (Jeresaty, Khan, and Landry, 1972). The patient reported here is unique in that he developed two separate manifestations of exquisite sensitivity to lignocaine: central nervous system toxicity, and cardiac toxicity in the form of extreme sinus bradycardia with a rate of 14 to $20 /$ minute. These events, furthermore, occurred in the absence of congestive heart failure, thus excluding the possibility of excessive blood levels. There has not been any evidence of 'sick sinus syndrome' or of AV block at any time during the subsequent follow-up period, either of which might have accounted for the effects of lignocaine on the cardiac electrical events. Finally, the possibility of inadvertent overdosage was also excluded. It is therefore not clear why this patient developed such a severe reaction to only $50 \mathrm{mg}$ lignocaine. One can only surmise, as mentioned earlier, an exceptional cerebral as well as cardiac sensitivity to lignocaine.

The question of possible potentiation of lignocaine by other drugs must be raised. The possibility that the oral dose of $750 \mathrm{mg}$ of procainamide the day before could have potentiated the effect of lignocaine is slight because of the short half life of this drug and the lapse of time between the last dose and the injection. The patient also received $2 \mathrm{mg}$ diazepam intravenously as anticonvulsant therapy. The time relation between the injection of diazepam and the onset of bradycardia could easily be construed as indicating a cause and effect relation. However, diazepam has not been reported, to the best of our knowledge, to produce sinus bradycardia, except in large doses in two dogs in one experimental study (Nevins, 1971). It should be noted, as mentioned previously by Zelis (1972), that three instances of severe sinus bradycardia so far noted after lignocaine have occurred in patients who were receiving other drugs as well (Jeresaty et al., 1972; Lippestad and Forfang, 1971 ; Wood, I97I). The possible additive effect of lignocaine and other drugs remains an unresolved question.

Reactions to lignocaine such as described here should not, however, be entirely unexpected in clinical situations for it is clear that some patients do exist who are extremely sensitive to lignocaine though it is admittedly difficult to produce sinus bradycardia with small doses of lignocaine in experimental animals (Lieberman et al., 1968) or man (Rydén, Cullhed, and Wasir, 1972). Lignocaine also exerts little electrophysiological effect on atrial fibres in tissue bath preparations (Mandel and Biggar, 197I).

The necessity for careful observation after lignocaine administration, already indicated by Cheng and Wadhwa (1973), can only be again emphasized. The need to administer lignocaine with extreme caution has become well recognized in patients of advanced age, or in those with sinus node dysfunction or inferior wall myocardial infarction. Continuous infusion of lignocaine in the presence of hepatic dysfunction or severe congestive heart failure is especially hazardous, since they predispose to excessive blood levels of lignocaine. This report, and others, of sensitivity to small doses of lignocaine suggests in addition that it may not be advisable to administer the drug prophylactically to all patients with suspected acute myocardial infarction as has been recently advocated.

\section{References}

Cheng, T. O., and Wadhwa, K. (1973). Sinus standstill following intravenous lidocaine administration. Fournal of the American Medical Association, 223, 790. 
Flensted-Jensen, E., and Sandøe, E. (1969). Lidocaine as an antiarrhythmic agent. Acta Medica Scandinavica, 185, 297.

Jeresaty, R. M., Kahn, A. H., and Landry, A. B. (1972). Sinoatrial arrest due to lidocaine in a patient receiving quinidine. Chest, 6r, 683.

Lieberman, N. A., Harris, R. S., Katz, R. I., Lipshutz, H. M., Dolgin, M., and Fisher, V. J. (1968). The effects of lidocaine on the electrical and mechanical activity of the heart. American fournal of Cardiology, 22, 375.

Lippestad, C.Th., and Forfang, K. (1971). Production of sinus arrest by lignocaine. British Medical fournal, I, 537.

Mandel, W. J., and Biggar, J. T., Jr. (I97I). Electrophysiologic effects of lidocaine on isolated canine and rabbit atrial tissue. Fournal of Pharmacology and Experimental Therapeutics, $\mathbf{1 7 8}, 81$.
Nevins, M. A. (197I). Ventricular arrhythmias and diazepam. (Letter to the Editor.) Fournal of the American Medical Association, 215, 643.

Parameswaran, R., Kahn, D., Monheit, R., and Goldberg, H. (I974). Sinus bradycardia due to lidocaine : clinical-electrophysiologic correlations. Fournal of Electrocardiology, 7, 75 .

Rydén, L., Cullhed, I., and Wasir, H. (1972). Effect of lignocaine on heart rate in patients with sinus bradycardia associated with proven or suspected acute myocardial infarction. Cardiovascular Research, 6, 664.

Wood, R. A. (I97I). Sinoatrial arrest: an interaction between phenytoin and lignocaine. British Medical fournal, I, 645.

Zelis, R. (1972). How benign is lidocaine? Chest, 61, 599.

Requests for reprints to Dr. Herman Klein, Meir General Hospital, Kfar-Saba, Israel.

\section{Addendum}

Since submission of our paper, another article (Parameswaran et al., 1974) has demonstrated the same effect in two patients, both of advanced age. 